\title{
FEV1/FVC Pre-Bronchodilator
}

National Cancer Institute

\section{Source}

National Cancer Institute. FEV1/FVC Pre-Bronchodilator. NCI Thesaurus. Code C159317.

The test for pre-bronchodilator forced expiratory volume over the forced vital capacity percentage value. 